\title{
Value of the electroencephalogram in viral encephalitis
}

\begin{abstract}
Background: The diagnosis and early treatment of infectious diseases of the Central Nervous System (CNS) are determinant for the prognosis of the patient, since a delayed, incorrect or insufficient treatment will result in mortality and irreversible sequelae. The electroencephalogram (EEG) continues to be the most accessible and economical diagnostic tool for the evaluation of CNS function, as it is a sensitive test to detect neuronal dysfunction.
\end{abstract}

Clinical Case: The main clinical manifestations, the results of the complementary exams, the results of the neuromonitoring by means of the EEG and the clinical evolution of an eight year-old patient diagnosed with herpetic viral encephalitis are described

Conclusions: Electroencephalographic monitoring offers great benefits in the evolutionary monitoring of infections of the CNS, allowing early detection of complications or relapses and the indication of sequelae or residual brain damage.

Keywords: electroencephalogram, viral encephalitis, neuromonitoring, children
Volume 8 Issue 6 - 2018

\author{
Liane Aguilar- Fabré,' René Francisco \\ Rodríguez-Valdés,' Rogelio Ridel Odales- \\ Ibarra, ${ }^{2}$ Ramiro Jorge García- García, ${ }^{3}$ Hebert \\ Luis Hernández- Montiel' \\ 'Department of Biomedical Research, Autonomous University \\ of Queretaro, Mexico \\ ${ }^{2}$ Department of neurology, Hospital of Pediatric Specialties, \\ Mexico \\ ${ }^{3}$ Neuropediatric Service, Pediatric Hospital, Mexico
}

Correspondence: René Francisco Rodríguez-Valdés, Clinic of the Nervous System, Department of Biomedical Research, School of Medicine,Autonomous University of Queretaro, Queretaro, Mexico, Carnation \# 200, Prados de la capilla, Queretaro, Queretaro, CP 76170, Mexico,

Email lafrrrv@yahoo.com

Received: August 17, 2018 | Published: November 16, 2018
Abbreviations: CNS, central nervous system; EEG, electroencephalogram; ICU, intensive care unit; CSF, cerebrospinal fluid; PCR, polymerase chain reaction; PCR, pleds, periodic lateralized epileptic form discharges; DNA, dexosyribonucleic acid

\section{Introduction}

The term encephalitis is used when there is clinical and/or pathological evidence of involvement of the cerebral hemispheres, brainstem or cerebellum due to an infectious process. Numerous microorganisms can produce symptoms and central neurological clinical signs through direct or indirect damage to the tissues of the Central Nervous System (CNS), including viruses. ${ }^{1}$

Viral infections to the CNS are usually benign and self-limiting with a good clinical evolution. However, a subgroup of viruses can result in a severe clinical course with high morbidity and mortality. ${ }^{2}$

Acute viral encephalitis is more common in children (more than 16 cases X 100000 patients per year) than in adults (3.5-7.4 cases X 100000 patients per year). Herpetic encephalitis is the most frequent viral encephalitis. The cause is herpes simplex virus type I in adults and type II in neonates. Therefore, if viral encephalitis is suspected, it will be treated as if it were a herpetic one until it is proven otherwise. ${ }^{2}$

The diagnosis and early treatment of infectious diseases of the CNS are decisive for the prognosis of the patient, since a delayed, incorrect or insufficient treatment will result in mortality and irreversible sequelae. The mortality of herpes simplex encephalitis is considerable and important neurological sequelae are reported among which are: cognitive deficit, mental retardation, personality changes, movement disorders, hemiparesis and seizures, among others (1 and 3).

The electroencephalogram EEG continues to be the most accessible and economical diagnostic tool for the evaluation of CNS function, as it is a sensitive test to detect neuronal dysfunction. Its performance in the form of neuromonitoring is able to provide valuable information to the diagnosis, evolution and possible sequelae in the infectious processes of the CNS.

\section{Clinical case}

An 8year-old male patient admitted to the Intensive Care Unit (ICU) of the Pediatric Hospital "Juan Manuel Marquez", Havana, Cuba, for presenting acute-setting neurological manifestations: disorders of consciousness and convulsions associated with fever, which led to suspicion from an infection of the CNS. No personal pathological or family history of interest is collected.

Upon physical examination the patient was obtunded, altered consciousness that rapidly evolved to coma, pyramidal signs were found such as spastic quadriparesis, osteotendinous hyperreflexia in all four extremities (Right $>$ Left), generalized hypertonia with bilateral equinism in the lower limbs, Babinsky sign bilateral and bilateral clonus of the ankles. The pupils were reactive to light.

The seizures began before 72 hours after admission and were characterized by being focal in the half of the left face, with spread to that hemi body and secondary generalization, very difficult to control, which evolved to an epileptic status and seizures resistant to antiepileptic treatment, which motivated a long stay in the ICU and later in the Neuropediatric service.

The initial analysis of the cerebrospinal fluid was normal (clear and transparent appearance, Leukocytes: 0, Pandy negative, Proteins: $0.14 \mathrm{~g} / 1$, Glucose: $3.2 \mathrm{mmol} / \mathrm{l}$ ) the culture was negative and the cerebrospinal fluid (CSF) for Herpes virus negative. The CSF study was repeated later and the viral DNA was confirmed by polymerase chain reaction (PCR). 
Axial Computed Tomography of the skull was performed, which showed a small ventricular system with no subarachnoid space and a decrease in density at the level of the generalized cerebral cortex. Based on the clinical findings the diagnosis of an acute infection to the CNS, viral encephalitis, was considered and an electroencephalographic evaluation was decided in order to corroborate the diagnosis.

Neuro monitoring was performed by (EEG), a total of 25 electroencephalographic assessments during the periods of installation, plateau and resolution of the infectious picture covering the entire clinical and electrophysiological evolution of the disease.

EEGs performed on the second and third day of evolution showed the presence of polymorphic generalized delta activity Figure 1. From the fourth day EEGs characterized by the appearance of periodic lateralized epileptiform discharges (PLEDs) that had their highest voltage over the fronto-temporal regions these complexes persisted for approximately 10days Figure 2. In the records made after the month of evolution, ictal electroencephalographic patterns are observed where the existence of an electro-clinical correlate with focal and focal seizures is secondarily generalized (by Video-EEG) confirming the diagnosis upon discharge of the patient. : Symptomatic partial epilepsy of difficult control, sequela of herpetic viral encephalitis Figure 3.

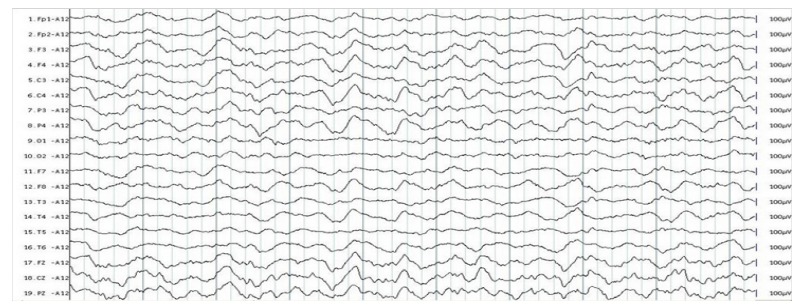

Figure I Electroencephalogram obtained after 3days of patient evolution where generalized polymorphous slow (delta) activity is observed.

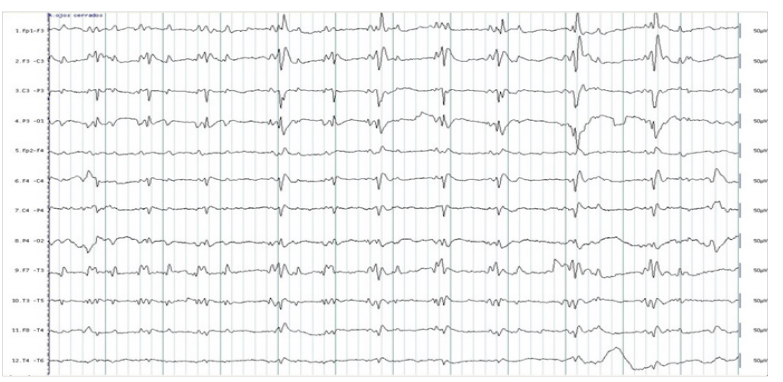

Figure 2 Periodical bilateral complexes are observed on temporal and frontal regions with left predominance.

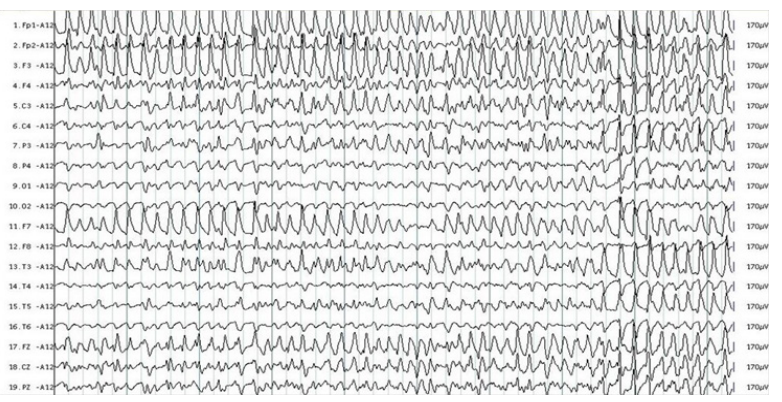

Figure 3 Electro-clinical focal crisis is secondarily generalized in the form of acute waves with maximum in left fronto-temporal regions.

\section{Discussion}

The most common neurological symptom of viral encephalitis in the acute phase is the disorder of consciousness manifested as: disorientation, confusion, drowsiness or coma, reported in up to $97 \%$ of cases, fever $(90 \%)$, headache $(81 \%)$, seizures $(67 \%)$, ataxia $(58 \%)$, aggressiveness (40\%), hemiparesis (33\%). ${ }^{1}$

The appearance of acute symptomatic seizures in the course of a viral encephalitis as occurred in this patient is a risk factor for the development of epilepsy and is associated with a worse prognosis. $50 \%$ of patients with a herpes simplex encephalitis make acute symptomatic crises which are justified by the participation in the infectious process of the highly epileptogenic temporo-frontal cortex. Therefore, encephalitis is the most frequently associated with epilepsy. ${ }^{3}$

With this case it is also evident that the association of encephalitis, a score on the Glasgow coma scale of 12 points or less and the presence of a neurological deficit has an important predictive value related to the appearance of acute symptomatic crises as shown by the literature. Suggesting that the greater the extent of parenchymal damage, the greater vulnerability to symptomatic crises. ${ }^{4,5}$

As it happened in this patient, it is reported that the initial CSF study can be normal up to $12 \%$ (2). CT scan of the skull showed a small ventricular system with absence of subarachnoid space, and attention is drawn to a decrease in density at the cerebral cortex level, which is generalized. As described in the literature reviewed, the tomographic findings may not be conclusive for this diagnosis; however magnetic resonance as a sensitivity of $86 \%{ }^{6}$

The first electroencephalographic reports in patients with CNS infectious diseases were made by Lindsley and Cutts in 1941, however it is not until 1959 that Millar and Coey describe the specific electroencephalographic alterations in herpes simplex encephalitis.?

In encephalitis the electroencephalographic findings are similar to those seen in meningitis, but the abnormalities are more severe, constituting a point of help in the differential diagnosis.?

As can be seen in Figure 1, the electroencephalographic records obtained on the second and third day of evolution show a disorganized base activity and the presence of diffuse polymorphic delta activity, which corresponds to the acute phase of this disease.

The degree of EEG slowing depends on the severity of the infection, the level of consciousness and other associated systemic and metabolic factors. As evidenced in this case, the earliest anomalies in acute encephalitis is the presence of diffuse slow activity in the high voltage delta range, demonstrating that the white matter of both cerebral hemispheres is compromised which explains the rapid evolution of consciousness disorder. ${ }^{7,8}$ From the fourth day of evolution of the disease in the ICU, electroencephalographic recordings are obtained characterized by the appearance of slow periodic angular wave complexes (PLEDs) that have their maximum expression on the fonto-temporal regions, these complexes persisted for approximately 10days, they are seen in Figure 2.

In this patient, the temporal evolution of the typical electroencephalographic pattern, that is, diffuse slow activity that evolves to PLEDs, with the involvement preferably of the temporal lobes and the basal part of the frontal lobes, points towards the diagnosis of a herpes virus encephalitis in spite of that the results of the CSF analysis initially and the CT scan were not conclusive for this 
diagnosis. ${ }^{7,8}$

The PLEDs in the course of viral encephalitis cannot be detected in some cases however, only when there are serial records as in this study is that these can be confirmed, reaffirming the usefulness of neuromonitoring of infectious diseases of the CNS since the admission of the patient in the ICU. ${ }^{7}$

Although these electroencephalographic findings are not pathognomonic of the disease, the presence of periodic bilateral complexes in association with fever and the rapid evolution of neurological signs are strong evidence for the diagnosis of herpes virus encephalitis. ${ }^{7,8}$ The EEG sensitivity for encephalitis is $90 \%$ higher than that reported for magnetic resonance studies. ${ }^{9}$ On the other hand, the presence of PLEDs in the electroencephalographic records before the diagnosis of viral encephalitis is closely related to the evolution and prognosis of the patient. ${ }^{10-12}$ The status epilepticus in this patient constitutes a risk factor for the later development of an epilepsy of difficult control. ${ }^{4-6,12}$ During the recordings made with the video-EEG in the neurophysiology service, the behavioral expression of the seizures and their electrical correlate was simultaneously recorded, contributing to a better characterization of them for a better management and treatment of them, expression of symptomatic focal epilepsy as a sequel to acute viral encephalitis. ${ }^{13}$

Although the realization of an EEG is not necessary to make the diagnosis of an infectious disease of the CNS, even with the advent of the new neuro imaging techniques the EEG continues to be a useful and innocuous tool for the study of the function of the brain's electrical activity as evidenced in this study. The herpes virus causes a necrosis usually severe in the areas mostly involved, destruction of the architecture, hemorrhage and in the most severe cases loss of all the glial and neuronal elements, you can find hemorrhage and necrosis foci on the temporal and frontal regions. Therefore, the mortality of this disease is considerable and approximately half of the survivors between 5 and 11years old have a significant residual deficit such as the symptomatic focal epilepsy of difficult control reported in this patient. $^{2}$

\section{Conclusion}

Neuro monitoring through electroencephalographic evaluations offers great benefits in the evolutionary monitoring of Central Nervous System infections, the early detection of complications or relapses and the indication of sequelae or residual brain damage.

\section{Conflict of interest}

The author declares there is no conflict of interest.

\section{References}

1. De Tiegè X, Rozenberq F, Hèron B. The spectrum of herpes simplex encephalitis in children. Eur J Paediatr Neurol. 2008;12(2):72-81.

2. Bernard LM, Bale FJ. Infections of the Nervous System. In: Menkes JH, et al. editors. Child Neurology. New York: Lippincott Williams \& Wilkins; 2006:433-556.

3. Misra UK, Tan CT, Kalita J. Viral encephalitis and epilepsy. Epilepsy. 2008;49(6):13-18.

4. Wang IJ, Lee PI, Huang LM, et al. The correlation between neurological evaluations and neurological outcome in acute encephalitis: a hospitalbased study. Eur J Paediatr Neurol. 2007;11(2):63-69.

5. Kim MA, KM Park, Kim SE, et al. Acute symptomatic seizures in CNS infection. Eur J Neurol. 2008;15(1):38-41.

6. Al-Sheklee A, Kocharian N, Suarez JJ. Re-evaluating the diagnostic methods in herpes simple encephalitis. Herpes. 2006;13(1):17-19.

7. Westmoreland BF. The EEG in cerebral inflammatory processes. In: Niedermeyer E, et al. editors. Electro encephalography. New York: Lippincott Williams \& Wilkins; 2005:323-338.

8. Lai CW, Gragasin ME. Electroencephalography in simple herpes encephalitis. J Clin Neurophysiol. 1988;5(1):87-103.

9. Fowler A, Stödberg T, Ericksson M, et al. Childhood encephalitis in Sweden: etiology, clinical presentation and outcome. Eur J Paeditr Neurol. 2008;12(6):484-90.

10. Chen YJ, Fang, PC, Chow JC. Clinical characteristics and prognostic factors of postencephalitic epilepsy in children. $J$ Child Neurol. 2006;21(12):1047-1051.

11. Lin JJ, Lin KL, Wang HS, et al. Analysis of status epileticus related presumed encephalitic in children. Eur J Paediatr Neurol. 2008;12(1):32-37.

12. Lin JJ, Lin KL, Wang HS, et al. Analysis of status epilepticus related presumed encephalitis in children. Eur $J$ Paediatr Neurol. 2008;12(1):32-37.

13. Hsieh WB, Chiu NC, Hu KC, et al. Outcome of herpes simplex encephalitis in children. J Microbiol Immunol Infect. 2007;40(1):34-38.

\section{Acknowledgements}

None. 